\title{
Analysis of Chinese herbal creams prescribed for dermatological conditions
}

\author{
F M Keane, S E Munn, A W P du Vivier, N F Taylor, E M Higgins
}

\begin{abstract}
Objective To determine whether Chinese herbal creams used for the treatment of dermatological conditions contain steroids.

Design 11 herbal creams obtained from patients attending general and paediatric dermatology outpatient clinics were analysed with high resolution gas chromatography and mass spectrometry. Setting Departments of dermatology and clinical biochemistry.

Main outcome measure Presence of steroid.

Results Eight creams contained dexamethasone at a mean concentration of $456 \mu \mathrm{g} / \mathrm{g}$ (range 64 to

$1500 \mu \mathrm{g} / \mathrm{g})$. All were applied to areas of sensitive skin such as face and flexures.

Conclusion Greater regulation needs to be imposed on Chinese herbalists to prevent illegal and inappropriate prescribing of potent steroids.
\end{abstract}

\section{Introduction}

Alternative therapies are becoming increasingly popular, particularly among patients with chronic skin disorders such as eczema which are not cured by standard treatment. Parents of children with eczema and adult patients tend to regard herbal remedies as natural plant derived products which are therefore without side effects. In particular, they believe them to be free of steroids, unlike many of the treatments prescribed by general practitioners or dermatologists. Oral Chinese herbal preparations have been the subject of discussion for some time and have been shown to be of benefit in eczema. ${ }^{12}$ In practice, however, Chinese herbalists often prescribe creams, either alone or in conjunction with the oral preparations.

We became interested in Chinese herbal medicine as several of our patients reported improvement of their eczema when using alternative treatments. Many returned to our clinic when they could no longer afford the herbs (the cost was up to $£ 35$ per week), with either an exacerbation of their eczema during herbal treatment or a request to continue their treatment on the NHS. We suspected that these herbal creams contained a steroid and so analysed some of them.

\section{Methods}

Ten patients submitted Chinese herbal cream for analysis (seven children, three adults). One adult was using two creams, giving a total of 11 for examination. Four patients were simultaneously taking oral preparations. Their ages ranged from 4 months to 36 years and there was no racial preponderance. Five different suppliers in south London were identified.

The cream was accurately weighed and then mixed with ethyl acetate and water by using a vortex. The phases were separated by centrifugation and the organic phase was filtered through phase separating paper. This was evaporated, usually leaving an oily residue which solidified on cooling.

The oil in the residue was removed by liquid-gel chromatography. A portion of the purified extract was analysed as a derivative on a gas chromatograph. Peaks were compared with known standard steroid peaks (figure). The identity of peaks was confirmed by mass spectrometry of standard dexamethasone and selected test creams.

\section{Results}

Ten of the 11 creams were white with an aromatic smell. Most containers were small glass pots, which were either unlabelled or labelled only with the name of the cream or supplier and frequency with which to apply the cream. Two pots were labelled with Chinese writing indicating the ingredients of the cream which included yellow lily, Radix astragali, Herba menthae, and Radix angelica pubescentis. The indications for prescribing the treatment were eczema in seven cases, scaly scalp in two, and eczema herpeticum in one case (table).

Eight of the 11 creams contained dexamethasone at a mean concentration of $456 \mu \mathrm{g} / \mathrm{g}$ (range 64 to $1500 \mu \mathrm{g} / \mathrm{g})$. The two bottles labelled with the ingredients did not contain dexamethasone. All the

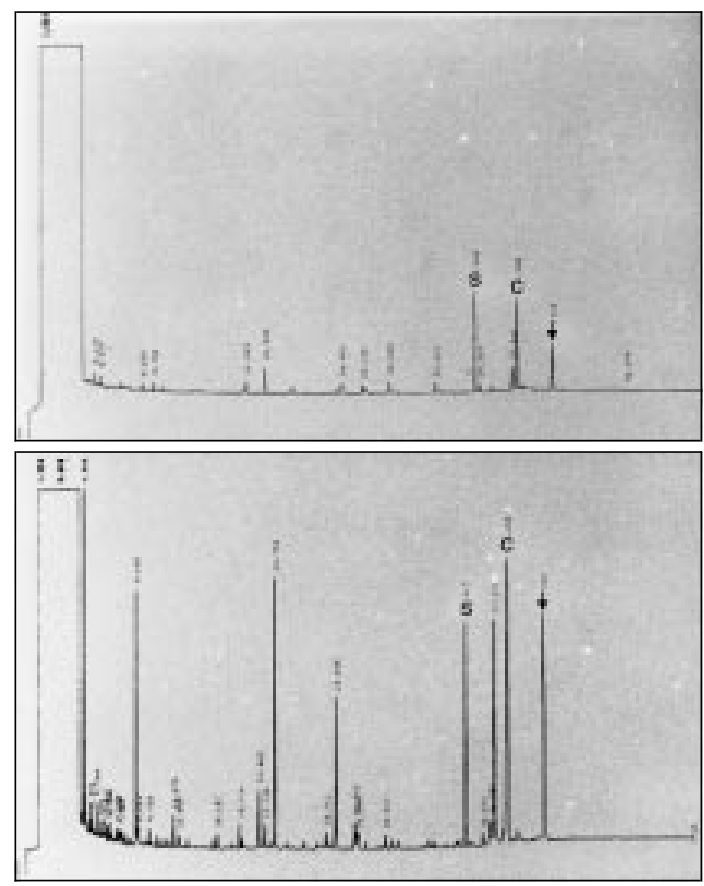

Gas chromatograms of standard cream containing dexamethasone (top) and Chinese herbal cream (bottom). $S=$ peak for internal standard 1, C=peak for internal standard 2, $\downarrow=$ peak for dexamethasone

\section{Departments of Dermatology and Clinical \\ Biochemistry, King's College Hospital, London SE5 9RS \\ F M Keane, specialist registrar in dermatology \\ S E Munn, senior registrar in dermatology \\ A W P du Vivier, consultant dermatologist N F Taylor, clinical biochemist E M Higgins, senior lecturer in dermatology \\ Correspondence to: Dr Keane FionaKeane99@ hotmail.com}

BMJ 1999;318:563-4 
creams prescribed for eczema (including 1 case of eczema herpeticum) contained dexamethasone. The concentration of dexamethasone in creams prescribed for children was 5.2 times higher than that in those prescribed for adults $(654.6 \mu \mathrm{g} / \mathrm{g} v 125 \mu \mathrm{g} / \mathrm{g}$ respectively). The highest concentration was prescribed for the face of a 4 month old baby with eczema.

All of our patients were unaware of the ingredients of the creams but had assumed that they did not contain steroids. As none had been given advice about potential side effects, the creams had been applied to the face and the flexures, often several times a day.

Dexamethasone content of Chinese herbal medicines prescribed for skin disorders and perceived effect

\begin{tabular}{lrll} 
Name of cream & $\begin{array}{c}\text { Dexamethasone } \\
(\mu \mathrm{g} / \mathbf{g})\end{array}$ & $\begin{array}{l}\text { Clinical } \\
\text { indication }\end{array}$ & $\begin{array}{l}\text { Clinical } \\
\text { effect }\end{array}$ \\
\hline Skinpro skin care lotion & 0 & $\begin{array}{l}\text { Eczema } \\
\text { herpeticum }\end{array}$ & Exacerbation \\
\hline Unlabelled & 60 & $\begin{array}{l}\text { Eczema } \\
\text { herpeticum }\end{array}$ & Exacerbation \\
\hline Unlabelled & 84 & Eczema & Improvement \\
\hline Pyong ping & 740 & Eczema & Improvement \\
\hline Yellow lily paste & 0 & Scaly scalp & No benefit \\
\hline Skin care No 1 & 227 & Eczema & Improvement \\
\hline Skin care No 1 & 334 & Eczema & Improvement \\
\hline Skin care No 1 & 460 & Eczema & Improvement \\
\hline Unlabelled & 0 & Scaly scalp & No benefit \\
\hline Ointment to ease inflamed skin & 1512 & Eczema & Improvement \\
\hline Unlabelled & 640 & Eczema & Improvement \\
\hline
\end{tabular}

\section{Discussion}

Dexamethasone is a prescription only, class II, potent steroid and its supply by unauthorised people is illegal in the United Kingdom. The mean dose of $456 \mu \mathrm{g}$ dexamethasone $/ \mathrm{g}$ cream is roughly equivalent to $0.05 \%$ betamethasone valerate, which is a more familiar preparation in the United Kingdom. The risk of side effects with such potent steroids is increased by their inappropriate use and application to areas of thin skin such as flexures and the face. We have no knowledge of the original source of the preparations or whether they all originated from the same supplier. The varying amounts of steroid found in each cream, however, suggests that some preparation and mixing occurred at each shop.

Chinese herbal medicines are widely available, but there is no governing body regulating practitioners or controlling their appropriate training. Concern about the arbitrary supply of herbs for oral use, the dose and content of which are apparently individually tailored, has already been voiced by dermatologists and hepatologists. ${ }^{3}$ The source and quality of the ingredients is also not standardised. Hepatotoxicity has been reported from oral preparations, and little is known about the long term toxicity of these medications. Sheehan et al have shown that standardised oral herbal preparations which are monitored in a conventional Western manner are beneficial in eczema. ${ }^{12}$ They also reported that these standardised preparations do not act like steroids. In our experience, however, most patients receive "personalised," unlabelled, and unstandardised preparations.

In 1990 it was suggested that the improvement noted during treatment with oral herbs might be due
- Patients with eczema often report improvement with Chinese herbal creams

- There may be no indication on the label about the contents of the cream

- Eight of the 11 creams analysed contained dexamethasone at concentrations inappropriate for use on the face or in children

- Inadvertent use of topical steroids can cause severe exacerbation of eczema herpeticum

- Closer regulation of herbal medicines is required

to a steroid in the cream which was concurrently prescribed. This theory, however, was never substantiated. ${ }^{5}$ Subsequently, the illegal supply of labelled oral ${ }^{6}$ and topical ${ }^{7}$ steroids by Chinese herbalists has been reported. We are concerned that our patients received both unlabelled and unlicensed topical treatment. Our results indicate that in many instances when patients receive combination oral and topical treatment, the topical element (which is essentially an unlicensed steroid) contributes to the clinical response. What must also be considered is the ethical issue arising from giving steroids to patients who, disillusioned with conventional treatment, believe they are taking herbal medications that do not contain steroids.

In conclusion, Chinese herbal creams are marketed, admittedly by word of mouth, as safe and steroid free. Although our sample numbers were small and represent only some south London outlets, most creams we tested, including all of those prescribed for eczema, contained steroids. Greater regulation and restriction needs to be imposed on herbalists, and continuous monitoring of side effects of these medications is necessary. The Medicines Control Agency is at present investigating these unofficial practitioners in close liaison with the newly opened Register of Chinese Herbal Medicine, which wishes to promote a uniform standard of good practice among traditional Chinese herbalists.

We thank Elizabeth V E M Okokon for help with the steroid analysis.

Contributors: FMK designed the project, collated the data, and wrote the paper. SEM helped in the design of the project and collection of creams for analysis. NFT performed the steroid analysis. EMH and AWPduV coordinated the research and are guarantors for the paper.

Funding: None.

Competing interests: None declared.

1 Sheehan MP, Rustin MHA, Atherton DJ, Buckley C, Harris DJ, Brostoff J, et al. Efficacy of traditional Chinese herbal therapy in adult atopic dermatitis. Lancet 1992;340:13-7.

2 Sheehan MP, Atherton DJ. A controlled trial of traditional Chinese medicinal plants in widespread non-exudative atopic eczema. BrJ Dermatol 1992;126:179-84.

3 MacGregor FB, Abermethy VE, Dahabra S, Cobden I, Hayes PC. Chinese herbs for eczema. Lancet 1989;299:1156-7.

4 Kane JA, Kane SP, Jain S. Hepatitis induced by traditional Chinese herbs; possible toxic components. Gut 1995;36:146-7.

5 Allen BR, Parkinson R. Chinese herbs for eczema. Lancet 1990;336:177.

6 O'Driscoll J, Burden AD, Kingston TP. Potent topical steroid obtained from a Chinese herbalist. Br J Dermatol 1992;127:543-4.

7 Hughes JR, Higgins EM, Pembroke AC. Dexamethasone masquerading as a Chinese herbal. Br J Dermatol 1994;130:261.

(Accepted 10 December 1998) 